Voix et Images

voixetimages

\title{
Sortir de la littérature
}

\section{Michel Biron}

Volume 28, numéro 1 (82), automne 2002

Noël Audet

URI : https://id.erudit.org/iderudit/000841ar

DOI : https://doi.org/10.7202/000841ar

Aller au sommaire du numéro

\section{Éditeur(s)}

Université du Québec à Montréal

\section{ISSN}

0318-9201 (imprimé)

1705-933X (numérique)

Découvrir la revue

\section{Citer cet article}

Biron, M. (2002). Sortir de la littérature. Voix et Images, 28(1), 166-171.

https://doi.org/10.7202/000841ar

Tous droits de reproduction, de rédaction et d'adaptation réservés 2002 C Université du Québec à Montréal
Ce document est protégé par la loi sur le droit d'auteur. L'utilisation des services d'Érudit (y compris la reproduction) est assujettie à sa politique d'utilisation que vous pouvez consulter en ligne.

https://apropos.erudit.org/fr/usagers/politique-dutilisation/
Cet article est diffusé et préservé par Érudit.

Érudit est un consortium interuniversitaire sans but lucratif composé de l’Université de Montréal, l'Université Laval et l'Université du Québec à Montréal. Il a pour mission la promotion et la valorisation de la recherche. https://www.erudit.org/fr/ 
moins, cet ouvrage émerge quelque peu du lot, tente de tisser des réseaux. Cependant, le rêve du passé est omniprésent. Des figures anciennes s'imposent à la conscience, s'opposent au déclin ambiant, réunissent les «particules élémentaires » avant leur dispersion ultime. Eschyle, Sophocle et Euripide (dans cet ordre, toujours) veillent sur le Québec, eux qui, en leur temps d'incertitude, avaient su si brillamment résister au déclin des valeurs et à la montée fulgurante de la complexité.
1. Michel Houellebecq, Les particules élémentaires, Paris, coll. «J'ai lu », 1998.

2. Joël de Rosnay, L'homme symbiotique. Regards sur le troisième millénaire, nouvelle édition, Paris, Seuil, 2000, p. 32.

3. Chantal Deschamps, Le chaos créateur, Montréal, Guérin, 2002, 168 p.

4. Marc Vaillancourt, Les feuilles de la sibylle. Défense de la littérature, Montréal, Trait d'union, 2002, p. 60 [139 p.].

5. Gilles Pellerin. La mèche courte. Le français, la culture et la littérature, Québec, L'instant même, 2001, p. 24 [140 p.].

\section{Roman}

\section{Sortir de la littérature}

\section{Michel Biron, Université du Québec à Montréal}

Louis Gauthier, Monique Proulx, Gérard Bouchard: trois romanciers, trois genres différents. L'un écrit un petit roman tourné vers l'intériorité du narrateur, l'autre raconte une fable sur le monde actuel, le troisième reconstitue le Québec de 1900 à partir d'une famille du Lac SaintJean. Roman d'une nouvelle subjectivité, roman ultracontemporain, roman historique : ce sont là, à bien $\mathrm{y}$ penser, trois orientations majeures $d u$ roman québécois actuel, si tant est qu'une classification comme celle-ci ait quelque sens aujourd'hui.

$$
* *
$$

Voyage au Portugal avec un Allemand ${ }^{1}$ de Louis Gauthier constitue, à mon sens, l'un des romans québécois les plus émouvants et les plus justes de l'année 2002, un des rares qui continue d'exister chez le lecteur une fois tournée la dernière page. Rien de recherché ou de complaisant dans ce livre au climat sombre, grave, rien pour épater le lecteur. En ce sens, c'est ce qu'on appelle un livre de maturité, dont on sent que l'auteur n'attend ni satisfaction, ni prestige, ni consolation. L'ambition proprement littéraire est absente, laissant la place à autre chose qui aurait très bien pu n'être rien du tout. Or, justement, ce roman parvient à tromper le rien à force d'y consentir.

Il y a un monde entre les calembours en tous genres des premiers romans de Louis Gauthier (depuis Anna en 1967) et la phrase simple 
de Voyage au Portugal avec un Allemand. Voici le début:

\begin{abstract}
Sans souci pour mon impatience, le train roule lentement dans la campagne française. Il s'arrête partout dans de petits villages qui vivent tranquillement à l'écart des capitales. Debout dans le couloir, n'ayant rien de mieux à faire, je fume cigarette sur cigarette en regardant défiler le paysage. C'est toujours la même chose: des arbres, des champs, des ponts, des rochers. Des rivières qu'on traverse. Des camions qui roulent sur des routes parallèles à la voie ferrée, puis qui disparaissent dans une courbe. Des cours de fermes, des arrières de maisons, des petits jardins. Des terrains de jeu où personne ne joue. Une autre gare. (p. 13)
\end{abstract}

On imagine difficilement un style plus descriptif, et pourtant, le ton est donné. Le coup d'œil, malgré la banalité apparente, est on ne peut plus personnel: c'est un homme impatient qui regarde. Devant lui, le monde bouge trop lentement et il est terriblement vide. Il y a des terrains de jeu, mais «personne ne joue». Lancé dans un voyage qui devrait éventuellement le conduire jusqu'en Inde, le narrateur se retrouve au Portugal. C'est à Lisbonne, dans un monde de transition où il ne connait personne, que ce narrateur choisit de s'arrêter.

Il finira bien par se passer quelque chose, se dit-il, comme s'il espérait que les hasards du voyage l'arrachent tôt ou tard à sa solitude, à sa peine d'amour, à sa peur de mourir. Comme on le devine par le titre, il rencontre à Lisbonne un Allemand, Monsieur Frantz, qu'il prend d'abord pour un prêtre. Son compagnon de route et de chambre est en fait un peintre, ou plutôt un ancien peintre. A présent, il n'a d'autre ambition que celle de s'installer quelque part au Portugal (il voudrait bien être cireur de soulier ou balayeur de rue). Philosophe du dimanche, il a l'air cruellement serein à côté du narrateur. Il parle de la mort, de la ville, des gens, puis termine ses phrases par une formule enfantine: «et pa pi pa po». Une sorte d'et cætera pour dire qu'ainsi va le monde.

La magie des mots a toutefois ses limites, constate le narrateur devant son petit carnet noir. Écrire quoi? «Le paysage n'a plus d'importance, le monde extérieur n'a plus d'importance, seule me préoccupe l'observation attentive du cratère qui s'ouvre en moi et dont la vue m'hypnotise.» (p. 49) Son moi est un immense trou qui lui donne le vertige: un néant, une Chose qui s'agrandit, se creuse et le laisse totalement angoissé, sans la moindre résistance. Il ne s'apitoie pas sur son sort, il n'écrit pas un récit sur soi, il n'attend rien des mots. Il avance dans la noirceur de cette effroyable solitude comme s'il allait disparaître bientôt. Pourquoi écrire? Les mots n'ont pas de poids face à la Chose, pas plus qu'Angèle, celle qui l'a abandonné et à qui il adresse des lettres qu'il n'enverra pas. Reste la marche, l'engourdissement du corps, une sorte d'abandon lucide à travers la douleur. C'est très précisément ce que parvient à raconter Louis Gauthier dans ce livre qui, à l'inverse de tant de romans contemporains «surécrits", ne dit pas un mot de trop. Leçon d'un écrivain qui sait de quoi il parle: «pour faire de la bonne littérature, il faut commencer par sortir de la littérature »(p. 113). 
Louis Gauthier n'est pas le seul écrivain à parler ainsi. On rencontre une idée similaire (bien qu'exprimée différemment) dans un des plus beaux livres parus récemment au Québec, Le sourire d'Anton ou l'adieu au roman d'André Major ${ }^{2}$. Les deux écrivains appartiennent à la même génération et ont participé de près à l'effervescence de la littérature québécoise, il y a plus d'un quart de siècle. Les deux ont choisi de s'éloigner du roman canonique avec lequel, de toute façon, ils n'avaient cessé de prendre mille libertés au profit du journal, du carnet, c'est-àdire au profit de formes qui renvoient à ce que Pavese (cité par Major) appelait le «métier de vivre». Il ne s'agit pas tant de préférer le décousu de l'écriture à la structure d'un genre fixe: l'art de Gauthier ou de Major est le contraire d'un abandon, d'un relâchement. La réussite d'un tel déplacement passe moins par le plaisir d'une écriture libre de contraintes que par la justesse d'une voix. C'est par là aussi qu'ils sortent de la littérature - et qu'ils y retournent comme si de rien n'était.

$$
* *
$$

Dans Le cœur est un muscle involontaire ${ }^{3}$ de Monique Proulx, c'est un peu l'inverse qui se produit: le roman se parfume à la littérature. On lit une sorte de chronique urbaine avec des personnages hypercontemporains empruntés à la culture locale et fascinés par la littérature. Le ton est léger, vif, très «deuxième degré », jamais «drabe ». Car le manque de désinvolture est un péché dans l'univers de Proulx. Les personnages ont tous quelque chose de doucement mystérieux, mais aussi de profondément ordinaire. C'est vous et moi, avec quelque chose en plus, une énergie, un désir de fiction. L'écriture refuse toutefois le grandiose et le pathétique: elle se veut jazzée, imprévisible, vivante, toujours tournée vers l'extérieur. La phrase nerveuse court dans tous les sens. Elle se donne à elle-même la réplique avant de rebondir vers le lecteur avec des clins d'œil; ensuite, nouveau coup de frein, changement de direction, elle invente des mots, agite un miroir et repart de plus belle non sans effectuer au passage quelques sorties de route. Si l'on veut se faire une idée du style et du rythme, on pensera à des chroniques journalistiques actuelles comme celles de Pierre Foglia ou de Jean Dion. (À chacun de juger s'il s'agit d'un compliment.)

Florence, la narratrice, une belle blonde pleine d'allant, gagne sa vie en créant des sites Web. C'est un métier original et surtout très neuf. Elle n'aime pas les écrivains - tous névrosés - et s'arrange habituellement pour ne pas les fréquenter. Malheureusement, Zéno, son patron et amant au prénom très littéraire (emprunté à l'écrivain italien Italo Svevo), appartient justement à la catégorie des névrosés. C'est aussi l'admirateur inconditionnel d'un écrivain appelé Pierre Laliberté, directement inspiré de Réjean Ducharme. On ne peut pas s'y tromper: trois citations en exergue, des titres avec motsvalises (Les récréatures, etc.), des pseudo-pastiches de Ducharme, la légende de l'écrivain qui fuit les médias, tout y est, sans parler de l'engouement de Zéno qui vire au culte pur et simple de l'écrivain fantôme ${ }^{4}$. 
Florence, lancée malgré elle sur la piste de Pierre Laliberté, ne résiste pas longtemps à la fascination. Mais, chez elle, les choses se compliquent considérablement et cela donne lieu à une quête d'un autre type. Elle, c'est un père qu'elle cherche.

Le roman s'ouvre, comme nombre de récits québécois récents ${ }^{5}$, sur la mort de son père véritable, appelé Pepa. À l'hôpital, Florence rencontre un écrivain déguisé en infirmier qui lui révèle les derniers mots du père: «le cœur est un muscle involontaire». Cette phrase structure toute l'intrigue $\mathrm{du}$ roman qui prend une allure vaguement policière à un certain moment, alors que Florence se lance à la poursuite de la sœur de Pierre Laliberté à New York. Elle emblématise surtout le personnage de Florence, car celle-ci fait de l'involontaire un art de vivre. Il ne faut pas beaucoup de temps pour comprendre où se trouve la blessure. La recherche désespérée d'un père, peu importe sa nature (biologique ou littéraire), a un je ne sais quoi de pathologique qui rend la narratrice plutôt attachante. Elle cherche quelqu'un qui, pour une fois, lui dirait quoi faire, comment agir, comment vivre.

La phrase du titre en dit plus long que ce qu'on croit entendre à la première lecture. Certes, c'est une phrase qui permet de faire le lien entre les deux figures paternelles, puisque Pepa l'aurait dite avant de mourir à Pierre Laliberté qui l'aurait ensuite placée à la fin de l'un de ses romans. Mais l'astuce aurait paru artificielle n'eût été de la portée plus large que Florence attribue à la phrase. C'est sa propre vie qu'elle place ainsi sous le signe de l'involontaire. Florence demande à Pierre Lali- berté (elle finit par le retrouver) de lui dire quelque chose, de lui enseigner au moins une vérité, comme si le romancier lui devait ça, après lui avoir volé les dernières paroles de son père. Elle se laisse conduire par la volonté du grand écrivain, devenant une sorte de terrain d'expérimentation ou une parfaite disciple. En témoigne la longue scène au cours de laquelle elle apprend à devenir curieuse grâce aux conseils techniques de Pierre Laliberté :

\section{Il propose que je choisisse deux individus parmi ces autres, que je les choisisse assez pour oublier mon existence à moi, que je leur donne la vie au détriment de la mienne. Le temps d'un exercice, une demi-heure tout au plus (p. 203).}

Et voilà Florence partie en quête de quelques autres qui deviendront elle durant une demi-heure. Mais il n'y a pas beaucoup de différence entre ces expérimentations limitées dans le temps et l'ordinaire de sa vie. Dès qu'elle change d'environnement, Florence s'abandonne à la volonté des autres: ce qui lui est étranger la séduit à tout coup. D'où une curieuse distorsion entre l'énergie $d u$ personnage et son pouvoir d'agir, entre la vie comme expérimentation continue et la pauvreté de chaque expérience vécue. Le quotidien de Florence est extraordinairement intense, pourtant il ne s'y passe pratiquement rien. La curiosité recherchée est censée la rendre vivante et présente dans le monde, mais elle ne lui permet de découvrir que des morts, des névrosés, des fantômes (Pierre Laliberté, Zéno, Pepa). À la fin, Florence se regarde écrire, à peine surprise de se découvrir semblable à ceux qu'elle prétendait détester: les 
écrivains. Elle a "envie d'écrire quelque chose qui débuterait ou se terminerait ainsi : «Recommençons, Florence. Recommençons. » De Ducharme, elle aura surtout retenu l'énergie du recommencement, la passion du départ.

$$
* *
$$

Il peut paraître surprenant de lire encore aujourd'hui des romans qui, à peu de choses près, ont l'air de sortir tout droit du dix-neuvième siècle. Et je ne parle pas du XIX ${ }^{e}$ siècle de Zola ou de Dickens, mais de notre dixneuvième siècle, celui de Jean Rivard et des Anciens Canadiens. C'est pourtant le cas du premier roman de l'historien Gérard Bouchard, Mistouk ${ }^{6}$, qui raconte la vie d'un jeune gaillard appelé Méo dans la paroisse de Mistouk, au lac Saint-Jean, dans les années 1900. «Méo le chat, Méo le héros!»Sa témérité fait damner sa mère et son père, la pieuse Marie et le bon Joseph, c'est une sorte de François Paradis qui aurait réussi à retrouver son chemin après s'être écarté. Il accumule les exploits sportifs (il est le premier à effectuer la traversée du lac à la nage), s'intéresse aux autos et aux bateaux, devient l'ami de Montagnais avec qui il passe l'hiver, travaille et voyage aux États-Unis, apprend à se méfier des Anglais qui possèdent les industries et les commerces, commet même un meurtre «politique » et se noie finalement en tentant d'échapper aux policiers.

Le personnage, on le voit, est taillé tout d'une pièce. Il appartient au bon vieux roman régionaliste, avec son franc-parler pittoresque (un «glossaire saguenayen » figure en annexe). Mais s'agit-il bien d'un personnage au sens fort du terme? Le romancier, confortablement installé dans les habits de l'historien et de l'ethnologue, s'en sert comme d'un prétexte pour brosser le tableau d'une société qu'il connaît mieux que quiconque. On apprend toutes sortes de choses sur les mœurs de l'époque, on rencontre Olivar Asselin, Louis Hémon, on comprend ce que fut la colonisation, le pouvoir de l'Église, etc. Est-ce assez pour faire un roman? Pour espérer sortir de la littérature, encore faudrait-il y être entré...

1. Louis Gauthier, Voyage au Portugal avec un Allemand, Saint-Laurent, Fides, 2002, 181 p.

2. André Major, Le sourire d'Anton ou l'adieu au roman, Montréal, Les Presses de l'Université de Montréal, 2001, 207 p.

3. Monique Proulx, Le cœur est un muscle involontaire, Montréal, Boréal, 2002, 401 p.

4. On peut se demander pourquoi Ducharme fait l'objet d'un tel culte dans la littérature québécoise récente, lui qui n'en demandait pas tant (un certain Hugo Roy a publié il n'y a pas longtemps, chez Boréal également, un roman-hommage sur Ducharme, intitulé L'envie). Imagine-t-on un tel hommage à un autre romancier de la Révolution tranquille? À Hubert Aquin, par exemple? Cela n'aurait pas le même sens. Aquin, c'est la révolution à l'ancienne manière, avec une cause et une utopie à la clef. Ducharme, c'est le rebelle sans cause, comme James Dean. Il incarne mai 1968 plutôt que la Révolution tranquille. La victoire de Ducharme sur Aquin dans l'imaginaire actuel permet de mieux voir ce qu'est devenue la littérature québécoise. Tandis qu'Aquin fait rêver ceux qui se souviennent d'une époque précise, Ducharme apparait comme l'expression d'une insaisissable violence. Or, c'est peut-être justement ce côté insaisissable qui fait de Ducharme un «père »de la littérature contemporaine.

5. Voir par exemple La petite fille qui aimait trop les allumettes de Gaétan Soucy (Boréal, 1998) ou la pièce Littoral de Wajdi Mouawad (Leméac/Actes Sud, 1999).

6. Gérard Bouchard, Mistouk, Montréal, Boréal, 2002, 510 p. 